\title{
TRATAMIENTO PALIATIVO DEL CÁNCER ESOFÁGICO Y DE LA UNIÓN GASTRO-ESOFÁGICA MEDIANTE COLOCACIÓN DE PRÓTESIS TRANSTUMORAL POR VÍA ENDOSCÓPICA*
}

\author{
Dr. Héctor Valladares H. ${ }^{1}$ \\ 1 Departamento de Cirugía Hospital Clínico Universidad de Chile. \\ Santiago, Chile.
}

TRABAJO DE INGRESO

\begin{abstract}
Esophageal stents for symptomatic treatment of esophageal cancer. Experience in 35 patients

Background: Esophageal cancer is usually diagnosed when it is beyond surgical ablation. Aim: To report the results of palliative treatment of dysphagia secondary to non-resectable esophageal carcinoma using self-expanding stents. Material and Methods: Review of medical records of 35 patients aged 57 to 94 years (21 males) with a non resectable esophageal carcinoma in whom a self-expanding stent was placed. Results: Eighty six percent of patients had severe dysphagia and it improved in all after the procedure. Two patients experienced a displacement of the stent. Three patients required the placement of a second stent. One patient died 30 days after the procedure due to pneumonia. Conclusions: Self expandable stents provide symptomatic relief to patients with non resectable esophageal carcinoma.
\end{abstract}

Key words: Esophageal cancer, stent, dysphagia.

\section{Resumen}

Objetivo: Mostrar método de paliación de disfagia por medio de prótesis autoexpandible, en pacientes con cáncer de esófago o unión esófago-gástrica en etapa IV, o considerados inoperables por patologías concomitantes severas. Material y Método: Estudio retrospectivo, 112 pacientes atendidos en nuestro hospital por cáncer de esófago, 43 en etapa IV se consideraron irresecables, de este grupo, en 8 se efectuó dilatación o gastrostomía endoscópica percutánea. Los otros 35 pacientes con disfagia de origen tumoral fueron tratados con prótesis esofágica como método paliativo al no resultar candidatos para tratamiento quirúrgico por estado de avance de enfermedad o sufrir patologías agregadas que la contraindicaban. Resultados: Tipo histológico más frecuente fue carcinoma epidermoide; nivel de disfagia fue grado 3 en mayoría de los pacientes $(86,4 \%)$, la que mejoró luego de la instalación de prótesis alcanzando nivel de disfagia grado $1(81,8 \%)$. Conclusiones: El uso de prótesis autoexpandibles es útil en el tratamiento de disfagia en pacientes con obstrucción de lumen esofágico secundario a crecimiento de tumor maligno.

Palabras clave: Disfagia, esófago, cáncer, paliación, prótesis.

*Recibido el 14 de agosto de 2014 y aceptado para publicación el 29 de marzo de 2015.

El autor no refiere conflictos de interés.

Correspondencia: Dr. Héctor Valladares H. hvalladares@gmail.com 


\section{Introducción}

El cáncer de esófago en Chile tuvo una tasa de mortalidad en el año 2011 de 4/100.000 habitantes (hombres 5,1 mujeres 2,8), con 1.038 egresos hospitalarios en año $2010^{1}$, similar a otros países europeos y sudamericanos. (Figuras 1 y 2). Presenta mal pronóstico con una sobrevida global a 5 años cercana al $10 \%^{2,3}$. Más del $50 \%$ de los pacientes son inoperables, lo que podría mejorar con tratamiento neoadyuvante ${ }^{4}$; aquellos que son operables usualmente resultan en una cirugía paliativa ${ }^{5-8}$. En Europa y Estados Unidos, a pesar de los avances aún con terapias neo-adyuvantes, los resultados no han mejorado significativamente, ya que en las mejores estadísticas la sobrevida a 5 años es de $40 \%$, lo que contrasta con experiencias en Japón ${ }^{9,10}$. En Chile la situación es bastante similar y la resecabilidad está en el rango de $30-60 \% 0^{11-13}$.

Por lo tanto, un grupo significativo de pacientes debe ser sometido a tratamientos paliativos, cuyo objetivo es mejorar en lo posible la sobrevida global y la calidad de vida, que en este caso se relaciona con la disfagia, entre ellos está la colocación de prótesis trans-tumorales, opción frecuentemente empleada, pero no libre de complicaciones ${ }^{14-17}$. En la Tabla $1 \mathrm{se}$ muestran las tasas de resecabilidad en oriente y occidente. En nuestro Departamento la tasa de resecabilidad actual es de $61 \%$ de los pacientes con indicación quirúrgica. De allí entonces la importancia que adquieren los tratamientos paliativos que significan un desafío para cirujanos, oncólogos, endoscopistas y radioterapeutas. Existe una gran variedad de opciones terapéuticas y hay considerable controversia sobre cuál usar, en forma única o combinada ${ }^{18-22}$. El rol de los procedimientos de bypass usando colon o estómago fue sugerido hace unas décadas, pero estaba asociado a alta morbi-mortalidad y escasa sobrevida. La braquiterapia ha sido sugerida para mejorar la disfagia pero no tiene un efecto inmediato en el tratamiento de esta ${ }^{3}$. La gastrostomía quirúrgica o percutánea, si bien permite alimentar al paciente, no es considerada una paliación eficiente pues no mejora la disfagia y más aún, los pacientes terminan
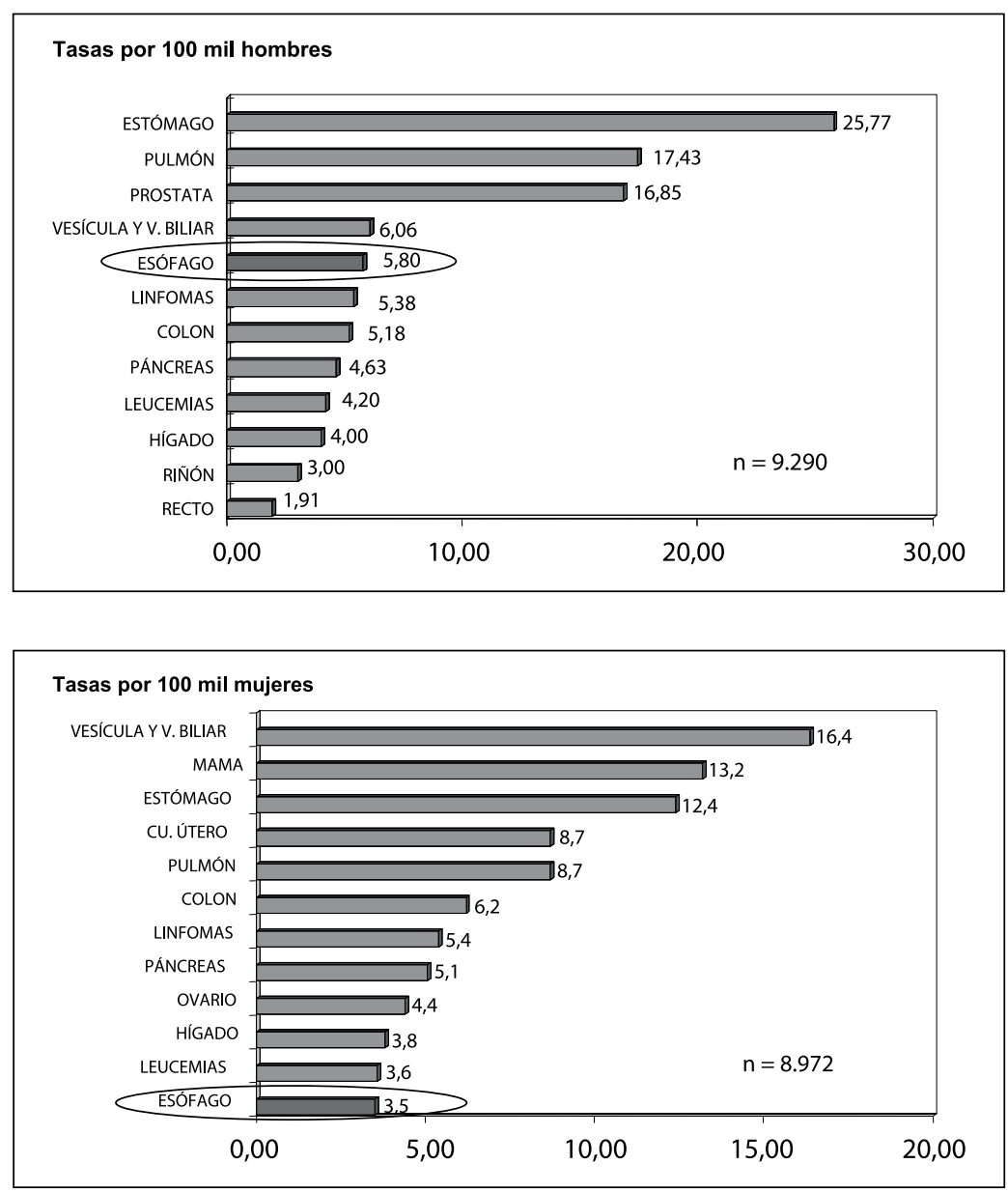

Figura 1. Tasas de mortalidad por cáncer en hombres (Minsal 2004).
Figura 2. Tasas de mortalidad por cáncer en mujeres (Minsal 2004). 
Tabla 1. Tasa de resecabilidad actual publicada

\begin{tabular}{|llll|}
\hline Autor & Año & País & Resecabilidad \% \\
Oriente & & & \\
$\quad$ Liu & 2004 & China & $96 \%$ \\
$\quad$ Puttawibull & 2001 & Tailandia & $36,1 \%$ (cirugía sola) \\
Occidente & & & $97 \%$ (terapia multimodal) \\
Nakadi & 2002 & Bélgica & $84 \%$ (cirugía sola) \\
& & & $18,2 \%$ \\
Dikken & 2013 & Inglaterra & $28,5 \%$ \\
& & Holanda & $41,45 \%$ \\
Universidad de Chile & 2009 & Dinamarca & $61,1 \%$ (cirugía sola y pacientes referidos) \\
\hline
\end{tabular}

en afagia presentando muchos de ellos cuadros de bronco-aspiración frecuentes. Para la mejoría de la disfagia se ha indicado desde hace décadas la dilatación para lesiones malignas, pero su efecto es temporal y requiere de dilataciones periódicas no estando exenta de riesgos de perforación, complicación que disminuyó su incidencia con el uso de dilatadores de balón o bien con el uso de dilatadores con guía, como los Savary y Eder-Puestow. Por todo lo anterior, una vez diagnosticada la irresecabilidad del carcinoma esofágico la terapia indicada en los últimos años ha sido la colocación de prótesis transtumoral con técnica de pull- through por vía endoscópica ${ }^{18}$.

El objetivo del presente trabajo es presentar nuestra experiencia en la colocación de prótesis auto expandible para el tratamiento paliativo de pacientes con cáncer de esófago inoperables.

\section{Material y Método}

\section{Pacientes estudiados}

En los últimos 5 años han sido evaluados en nuestro Departamento un total de 112 pacientes portadores de cáncer de esófago, de los cuales 43 pacientes $(38,9 \%)$ o se catalogaron en etapa IV irresecables (T4N1M1) o eran pacientes muy añosos por lo que fueron rechazados para tratamiento quirúrgico resectivo. De estos 43 pacientes, 8 (26,6\%) no se operaron por presentar lesiones tumorales con compromiso extra-esofágico, más adenopatías y/o metastasis a distancia y fueron sólo sometidos a tratamiento con dilataciones o gastrostomía endoscópica percutánea; 35 pacientes $(73,3 \%)$ fueron sometidos a tratamiento paliativo mediante la colocación de una prótesis transtumoral por vía endoscópica, constituyen el grupo de estudio incluido en este trabajo. Ellos corresponden a 21 hombres y $14 \mathrm{mu}-$ jeres con edad promedio de 73 años (rango 57-94). Todos estos pacientes fueron evaluados de acuerdo a nuestro protocolo de estudio con endoscopia, his- tología, examen radiológico baritado para estudio de localización y extensión axial del tumor, además de estadificación con tomografía computada de tórax y abdomen. En algunos casos se complementó con un PET scan por sospecha de enfermedad diseminada. En estos pacientes se descartó la cirugía por ser tumores irresecables o fueron excluidos ya sea por edad avanzada o por patología médica asociada, fundamentalmente cardiovascular y respiratoria, o por invasión a órganos vecinos. En las Figuras 3, 4 y 5 se muestran algunos ejemplos de grandes lesiones tumorales, incluso con fístula esófago-bronquial, en los cuales se indicó algún procedimiento paliativo.

Todos los pacientes o sus familiares dieron su consentimiento informado para colocación de prótesis, debido a su progresiva disfagia. Esta revisión fue aprobada por comité de ética de nuestro Hospital.

Los 35 pacientes presentaban diversos grados de disfagia, la que se clasificó de acuerdo al siguiente puntaje evaluado por el médico al momento de la anamnesis:

$\begin{array}{lll}\text { Puntaje } 0 & : & \text { Sin disfagia } \\ \text { Puntaje 1 } & : & \text { Ingesta de alimentos sólidos } \\ \text { Puntaje 2 } & : \text { Ingesta de semisólidos } \\ \text { Puntaje } 3 & : & \text { Ingesta sólo de líquidos } \\ \text { Puntaje } 4: & \text { Afagia }\end{array}$

\section{Método de colocación}

\section{Dilatación endoscópica pre colocación de prótesis}

Después de pre-medicación con anestesia tópica y sedación profunda bajo control por anestesiólogo si el caso lo requería, se practica endoscopia y se localiza la zona estenótica la que es franqueada por un alambre guía hacia distal bajo control fluoroscópico. Luego de esto se introduce en forma progresiva las bujías de Savary en diámetros progresivos nunca aumentando en más de 3 números consecutivos, o para lograr un diámetro aceptable de $36 \mathrm{~F}$. 


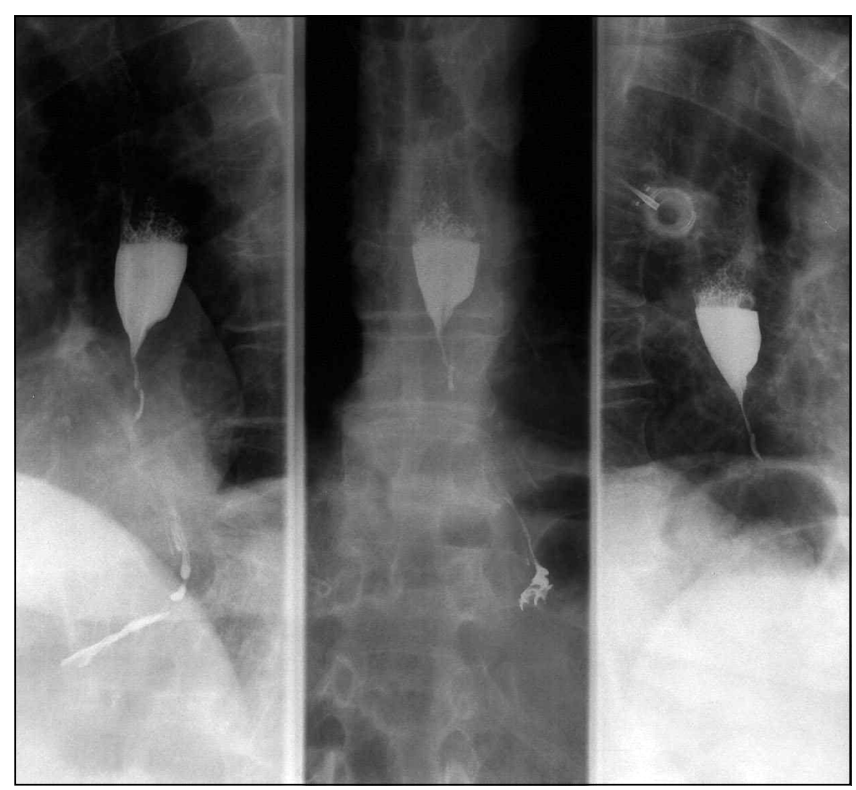

Figura 3. Cáncer esofágico extenso de tercio inferior irresecable. (T4).

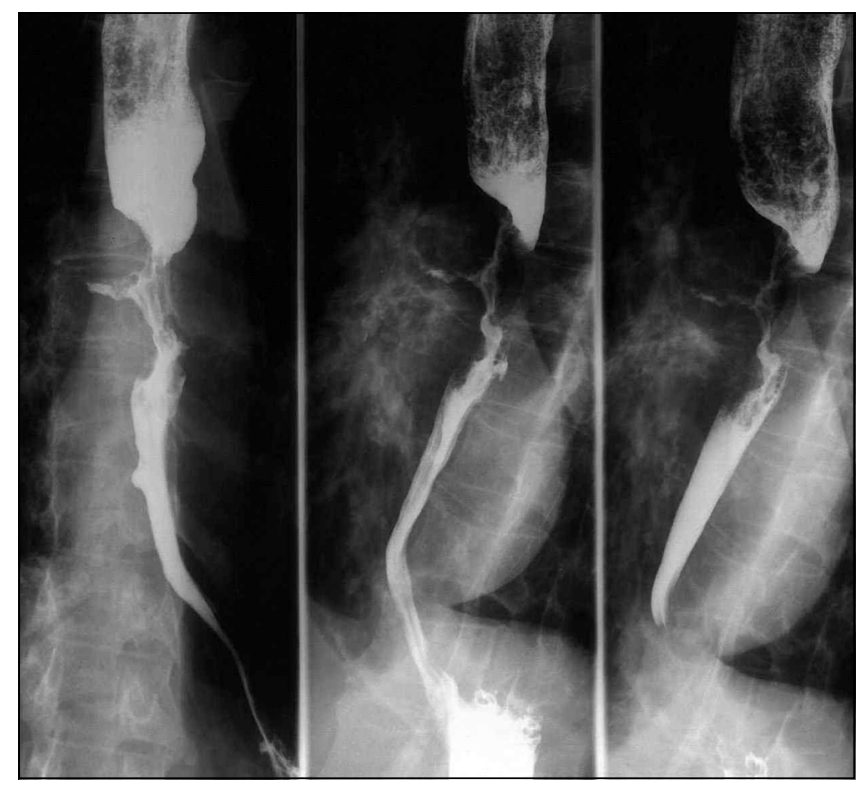

Figura 4. Cáncer de esófago de tercio medio fistulizado irresecable (T4).

\section{Colocación de prótesis transtumoral por vía endoscópica}

En nuestro Departamento este procedimiento se efectúa en pabellón quirúrgico bajo anestesia general, con apoyo radioscópico para marcación externa de los límites macroscópicos de la lesión tumoral, para determinar la longitud de la prótesis a instalar, y finalmente se efectúa la colocación bajo control radiológico y luego su revisión por vía endoscópica.
Se han utilizado 3 tipos de prótesis (Poliflex ${ }^{\circledR}$ de Boston Scientific, Z stent $\AA$ Cook Medical, Tecnostent ${ }^{\circledR}$ de Choostent).

En las Figuras 6, 7 y 8 se muestran las etapas del procedimiento de dilatación inserción y cómo queda insertada la prótesis una vez de retirado el dispositivo de inserción.

En la Figura 9 se muestra el control radiológico al día siguiente de instalada la prótesis. 


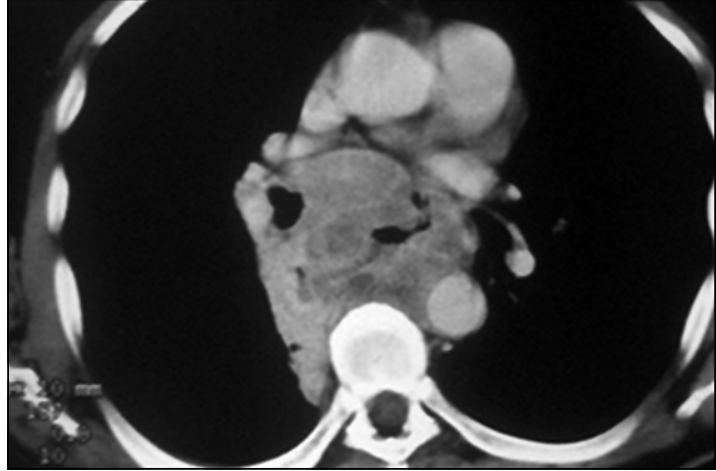

Figura 5. Tomografía computarizada de paciente con tumor esofágico irresecable sometido a colocación de prótesis transtumoral.

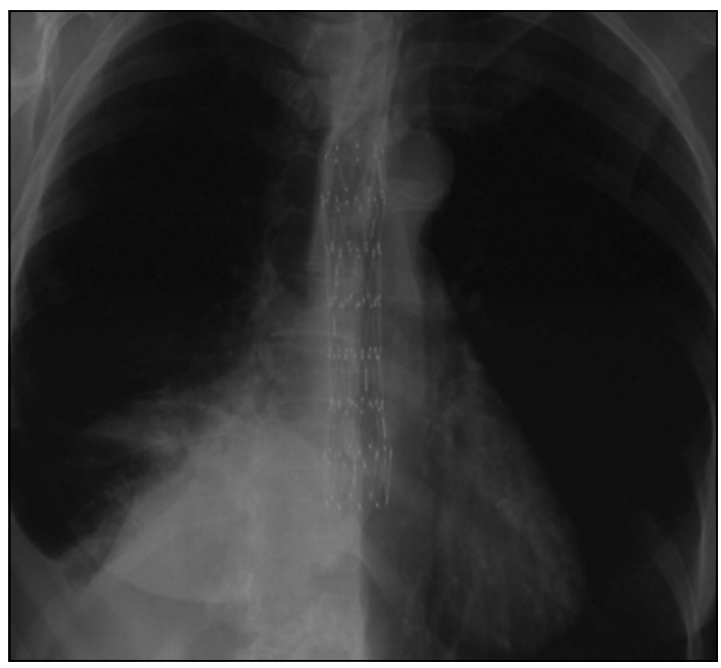

Figura 7. Prótesis auto expandible: posición final.

Los tipos de stent utilizados en este estudio fueron fundamentalmente prótesis metálicas, de nitinol, auto expandibles recubierta con silicona o poliuretano.

\section{Análisis estadístico}

Para el análisis estadístico, se usaron el Test de Wilcoxon y de Kaplan Meier para el cálculo de sobrevida con software Microsoft Excel.

\section{Resultados}

Las características demográficas y las características de la lesión tumoral se muestran en la Tabla 2. El tipo histológico más frecuente fue el carcinoma

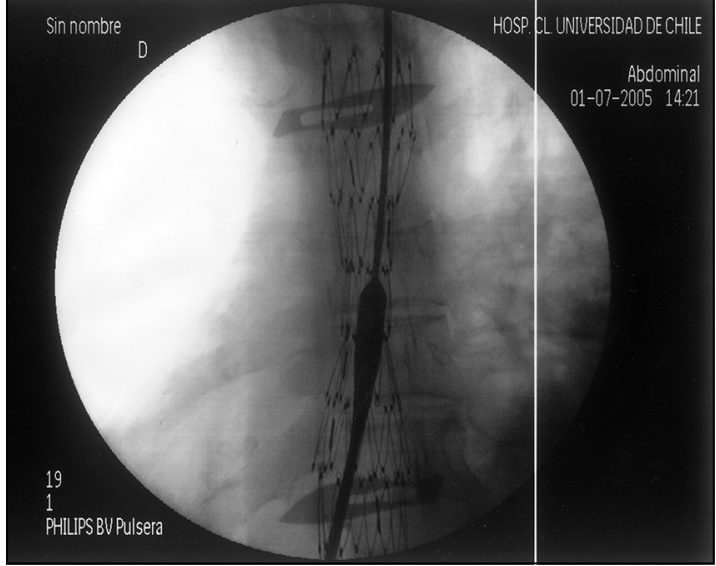

Figura 6. Colocación de prótesis intraluminal.

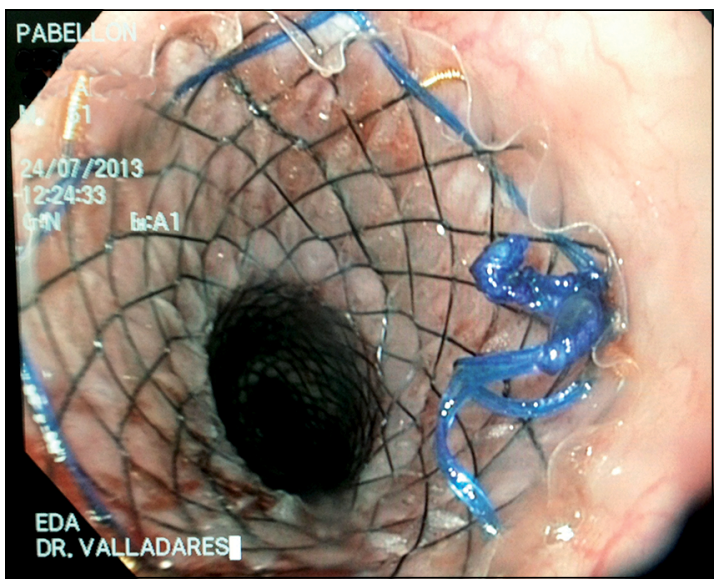

Figura 8. Control endoscópico al final de la colocación de la prótesis.

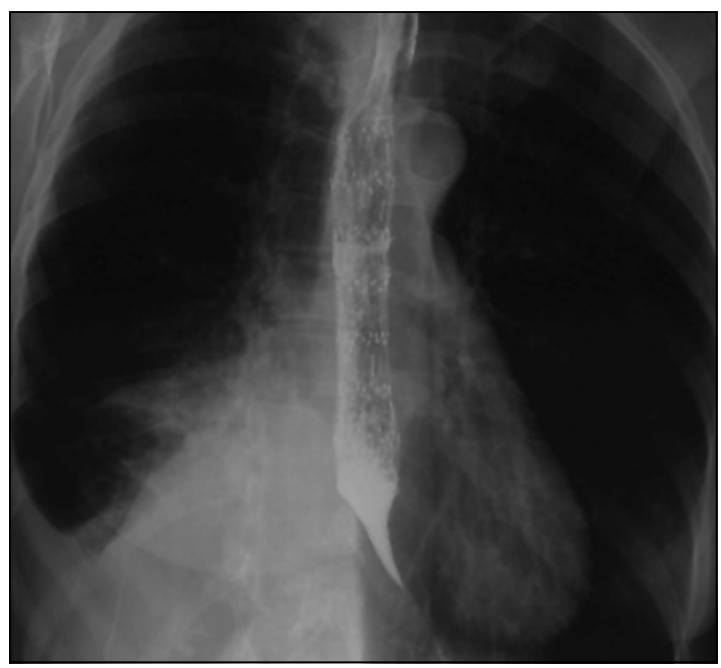

Figura 9. Control con tránsito baritado.

Rev Chil Cir. Vol 67 - No 4, Agosto; pág. 360-370 
epidermoide localizado en tercio medio. Los pacientes fueron catalogados en estadio IV por presentar lesiones T4, con invasión a estructuras vecinas o metástasis ganglionares.

La gran mayoría de los pacientes presentaban disfagia en categoría 3 (27 pacientes) previo al procedimiento.

La evolución clínica inmediata post procedimiento se muestra en la Tabla 3. No hubo complicaciones intraoperatorias y el tiempo total de la intervención, excluyendo la endoscopía y dilatación previa fue de $45 \pm 14$ min. El primer día después de instalada la prótesis el paciente se mantiene con régimen cero, analgésicos e hidratación parenteral con el fin de observar la evolución inmediata postoperatoria y descartar complicaciones respiratorias, y eventual perforación inadvertida. En 1 paciente se observó desplazamiento precoz de la prótesis, la que debió ser reposicionada por endoscopía. Este mismo paciente presentó posteriormente una neumonía con insuficiencia respiratoria, por lo que falleció antes de los 30 días; no hubo mortalidad relacionada al

Tabla 2. Pacientes incluidos y sus características clínicas

\begin{tabular}{|c|c|}
\hline Total & 35 \\
\hline Edad & $\begin{array}{l}\bar{X}=73 \\
\text { (rango 57-94 años) }\end{array}$ \\
\hline Sexo & $\begin{array}{l}\text { M: } 21 \\
\text { F: } 14\end{array}$ \\
\hline $\begin{array}{l}\text { Tipo histológico } \\
\text { Escamoso } \\
\text { Adenocarcinoma } \\
\text { Mesotelioma } \\
\text { Metástasis mediastínica Ca mama }\end{array}$ & $\begin{array}{r}23 \\
10 \\
1 \\
1\end{array}$ \\
\hline $\begin{array}{l}\text { Localización } \\
1 / 3 \text { medio } \\
1 / 3 \text { inferior } \\
\text { Con invasión de cardias }\end{array}$ & $\begin{array}{r}18 \\
10 \\
7\end{array}$ \\
\hline $\begin{array}{l}\text { Largo de lesión } \\
\quad<5 \mathrm{~cm} \\
\quad 5,1-8 \mathrm{~cm} \\
>8,1 \mathrm{~cm}\end{array}$ & $\begin{array}{r}6 \\
14 \\
15\end{array}$ \\
\hline $\begin{array}{l}\text { Disfagia } \\
\qquad \begin{aligned} 0 & = \\
1 & = \\
2 & = \\
3 & = \\
4 & =\end{aligned}\end{array}$ & $\begin{array}{r}0 \\
0 \\
8 \\
27 \\
0\end{array}$ \\
\hline $\begin{array}{l}\text { Tipo de lesión } \\
\text { Estenosante } \\
\text { Estenosante ulcerado } \\
\text { Estenosante perforado (fístula) }\end{array}$ & $\begin{array}{r}28 \\
2 \\
5\end{array}$ \\
\hline
\end{tabular}

Tabla 3. Resultados postoperatorios inmediatos: evolución clínica

\begin{tabular}{|c|c|}
\hline $\begin{array}{l}\text { Días de estada } \\
\text { intra-hospitalaria }\end{array}$ & $\begin{array}{l}\text { promedio } 3 \text { días } \\
\text { (rango 3-21 días) }\end{array}$ \\
\hline Realimentación oral & $2^{o}$ día \\
\hline \multicolumn{2}{|l|}{ Complicaciones inmediatas } \\
\hline $\begin{array}{ll}\text { Dolor } & \text { Leve } \\
& \text { Severo }\end{array}$ & $\begin{aligned} 33 & (94,28 \%) \\
2 & (5,71 \%)\end{aligned}$ \\
\hline Apertura de la estenosis & $\begin{array}{l}34 \text { ( } 1 \text { caso apertura } \\
\text { parcial mesotelioma) }\end{array}$ \\
\hline Mejoría de la disfagia & 34 \\
\hline Sellado de fístula & $5(100 \%)$ \\
\hline Hemorragia & 0 \\
\hline Perforación & $\begin{array}{l}2(5,71 \%) \text { (durante dila- } \\
\text { tación } 1, \text { al } 14^{\circ} \text { día) }\end{array}$ \\
\hline Desplazamiento precoz & $\begin{array}{l}1 * \text { reposicionamiento } \\
\text { endoscópico }\end{array}$ \\
\hline Complicación médica & $\begin{array}{l}12,8 \%) \text { (insuficiencia } \\
\text { cardíaca, mesotelioma) }\end{array}$ \\
\hline Mortalidad antes de los 30 días & $1 *$ (neumonía) \\
\hline Derivada del procedimiento & 0 \\
\hline
\end{tabular}

*Mismo paciente.

procedimiento mismo. Las complicaciones se muestran en la Tabla 7.

$\mathrm{Al}$ día siguiente de la colocación de la prótesis todos los pacientes fueros sometidos a control radiológico con bario para observar la dinámica de la progresión del bolo baritado y confirmar la posición definitiva de la prótesis (Figura 7). En 22 pacientes se confirmó correcta posición del stent y buen paso del bolo baritado. En 5 pacientes con sospecha de fístula bronco-esofágica establecida, se confirmó oclusión completa de la fístula. Post procedimiento se observó dolor, que en general fue leve, más bien una sensación de malestar retro esternal, 1 paciente presentó dolor más severo que fue manejado con analgesia intravenosa. Un paciente presentó perforación durante la dilatación previa a la colocación de prótesis que evolucionó satisfactoriamente con la colocación de la misma. No hubo complicaciones graves, tales como hemorragia, mediastinitis o perforación por la prótesis ni mortalidad derivada del procedimiento. La mejoría de la disfagia se obtuvo en todos los pacientes. La gran mayoría fueron dados de alta entre el $2^{\circ}$ y el $4^{\circ}$ día postoperatorio, uno de ellos presentó una insuficiencia cardíaca por lo que permaneció en el hospital durante 21 días. Un paciente falleció dentro de los primeros 30 días por 
Tabla 4. Evolución de la disfagia antes y después de la colocación de la prótesis

\begin{tabular}{|ccc|}
\hline Puntaje & Antes & Después \\
0 & 0 & 0 \\
1 & 0 & $31(81,8 \%)$ \\
\hline 2 & 8 & 3 \\
3 & $27(86,4 \%)$ & 1 \\
\hline 4 & 0 & 0 \\
\hline
\end{tabular}

una neumonía; correspondió al paciente en quien debió reposicionarse la prótesis, comprobándose al $3^{\text {er }}$ día del procedimiento una extensa neumonía con insuficiencia respiratoria en paciente con metástasis pulmonares y daño pulmonar crónico.

El costo total asociado al procedimiento varió entre \$ 1.474.000 (incluyendo costo de prótesis entre $\$ 450.000-\$ 650.000)$ en casos de cirugía electiva, y \$3.245.351 en un caso de colocación de prótesis de urgencia por tumor esofágico perforado.
En la Tabla 4 se muestra la evolución postoperatoria inmediata de la disfagia después de la colocación de la prótesis. Todos los pacientes mejoraron significativamente de su disfagia evaluada en los primeros días post colocación de prótesis, el $86,4 \%$ de los pacientes presentaban disfagia grado 3 antes del procedimiento, el $81,8 \%$ de los pacientes, posterior a este quedaron en disfagia grado $1(p=0,0)$.

En la Tabla 5 se muestran los resultados postoperatorios tardíos post colocación de la prótesis. Dos pacientes presentaron desplazamiento de la prótesis al $2^{\circ}$ mes de colocada y de iniciado tratamiento con radioterapia, la que al necrosar el tumor, ocasionó un desplazamiento del stent al estómago, la que fue retirada por vía endoscópica. En el segundo paciente la prótesis se desplazó sin causa aparente al estómago y fue retirada por vía quirúrgica, colocándose una segunda prótesis, pero el paciente fallece por neumonía aspirativa.

Treinta y dos pacientes se sometieron a colocación de 1 dispositivo, 3 pacientes debieron ser sometidos a colocación de una segunda prótesis $(8,5 \%)$ por complicaciones de la primera.

Tabla 5. Evolución tardía: complicaciones

\begin{tabular}{|lll|}
\hline Complicaciones & n & Tratamiento \\
\hline Reestenosis tumoral (por encima) & 2 & $\begin{array}{l}\text { Dilatación, resección parcial } \\
\text { Colocación de segunda prótesis }\end{array}$ \\
Desplazamiento & 2 & $\begin{array}{l}\text { Extracción endoscópica y recolocación de prótesis } \\
\text { Disfunción protésica }\end{array}$ \\
Disfagia (impactación de alimentos) & 2 & (colapso antero lateral dilatación endoscópica) \\
Reflujo gastroesofágico & 6 & Extracción endoscópica \\
Total & $13 / 35(37,2 \%)$ & \\
\hline
\end{tabular}

Tabla 6. Sobrevida de los pacientes tratados con colocación de prótesis auto expansibles $(n=35)$

\begin{tabular}{|llr|}
\hline Sobrevida & n \\
$<3$ meses & 14 \\
$3,1-6$ & 10 \\
$6,1-12$ & 7 & \\
$>12,1$ & $4 \quad$ (2 pacientes con prótesis sobre \\
\multicolumn{3}{|c|}{ prótesis) } \\
Media 4,3 $\pm 2,4$ meses (Rango 1-23 meses) & \\
Causas de muerte & Insuficiencia cardíaca & 1 \\
& Perforación y sepsis & 1 \\
& Neumonía aspirativa & 1 \\
& Infarto del miocardio & 1 \\
& Progresión de la enfermedad & 31 \\
\hline
\end{tabular}

Tabla 7. Resumen de las complicaciones actuales post colocación de prótesis transtumoral por vía endoscópica

\begin{tabular}{|lcc|}
\hline Complicaciones & $\mathbf{\%}$ & Referencia \\
\hline Hemorragia & $8-13 \%$ & $(26,27)$ \\
Ulceración & $8,3 \%$ & $(26)$ \\
Perforación & $1,9 \%$ & $(27)$ \\
Migración & $6-20,5 \%$ & $(24,25,26,30)$ \\
Dolor & $90-100 \%$ & $(15,21-24)$ \\
Complicaciones respiratorias & $2,6 \%$ & $(26)$ \\
Fístula & $5,1-6 \%$ & $(15-26,27)$ \\
Compresión vía aérea & $2,6-14,9 \%$ & $(26-27)$ \\
\hline Obstrucción & $8-12,5 \%$ & $(24,25)$ \\
\hline
\end{tabular}




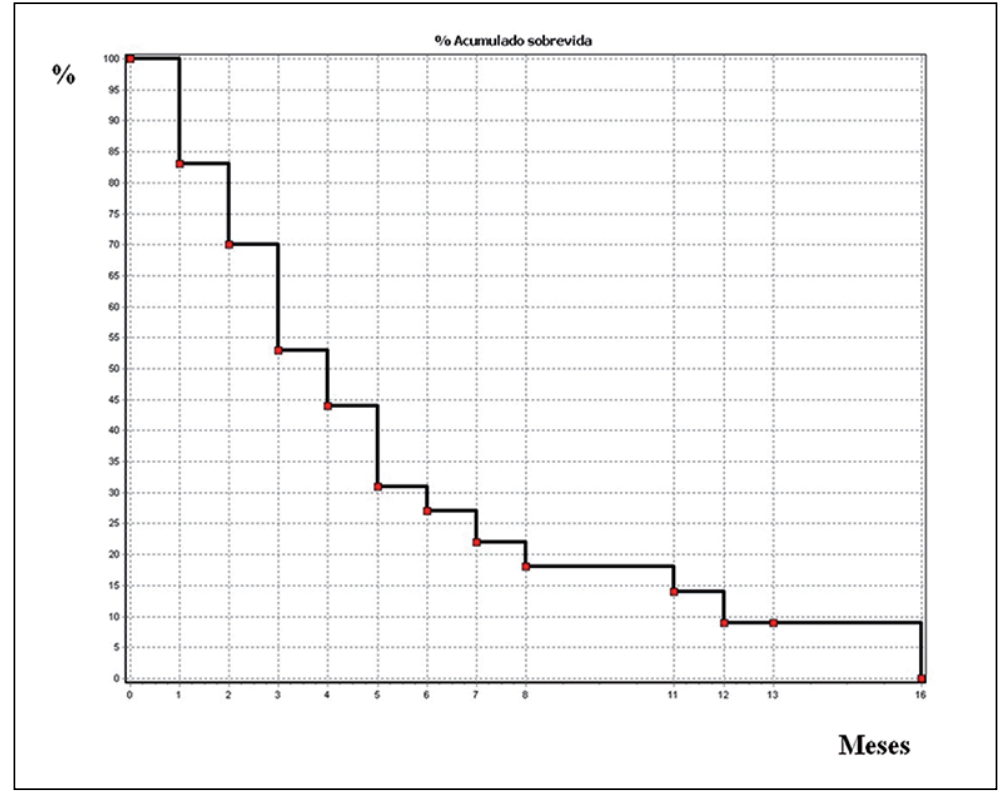

Figura 10. Curva de sobrevida en pacientes con cáncer de esófago sometidos a tratamiento paliativo con colocación de prótesis transtumoral.
Re-estenosis proximal del esófago por crecimiento tumoral se observó en otros 2 pacientes, siendo tratados con dilatación y resección parcial tumoral con asa diatérmica y en 1 de ellos recolocación de una segunda prótesis. La disfunción protésica, fue sospechada clínicamente y confirmada mediante estudio radiológico o endoscópico El manejo fue médico en 1 paciente $(2,8 \%)$, endoscópico en 4 pacientes $(11,4 \%)$ y quirúrgico en 1 paciente $(2,8 \%)$. Disfunción protésica a pesar de estar en buena posición se observó en 1 paciente por colapso antero lateral de la misma, la paciente se sometió a dilatación endoscópica con mejoría parcial de la disfagia quedando en grado 2. Atascamiento por alimentos sólidos también se observó al no cumplir las indicaciones dietéticas por parte de los pacientes, debiéndose extraer trozos de alimentos sólidos, con prótesis permeables; 4 pacientes presentaron reflujo gastro-esofágico que se manejó con inhibidores de la bomba de protones.

Aquellos casos en los cuales se confirmó obstrucción de la prótesis por tumor, fueron sometidos a manejo endoscópico para permeabilizar el stent mediante dilatación en 1 paciente, coagulación argón plasma en 1 paciente o colocación de una segunda prótesis en 2 pacientes.

En la Tabla 6 se muestra la sobrevida observada. Un caso falleció precozmente post procedimiento por insuficiencia respiratoria con metástasis pleurales y pulmonares. En este paciente se confirmó buena posición de la prótesis y buen funcionamiento.

La sobrevida media es de 4,3 $\pm 2,4$ meses con un rango de 1 a 23 meses. Siete pacientes tuvieron una sobrevida menor de 3 meses, 6 pacientes vivieron entre 3,1 y 6 meses, 5 pacientes vivieron entre 6,1 y 12 meses y los otros 3 pacientes entre 12 y 23 meses de sobrevida ( 2 de ellos con colocación de $2^{\mathrm{a}}$ prótesis), falleciendo por progresión de la enfermedad metastásica, pero usando su prótesis hasta su deceso. En la Figura 10 se muestra la curva de sobrevida usando el método de Kaplan-Meier.

\section{Discusión}

El cáncer de esófago en Chile, al igual que en el mundo occidental, sigue siendo un problema no resuelto ya que sólo un 30-35\% de los pacientes pueden ser sometidos a tratamiento quirúrgico resectivo, lo que en una gran proporción resulta en un tratamiento paliativo, siendo la sobrevida a 5 años de $30-40 \%$, lo que contrasta con experiencias en Japón. Actualmente hay muchas líneas de tratamiento que no han cambiado los resultados finales. Los pacientes consultan tardíamente con disfagia severa cursando un progresivo deterioro en la nutrición y calidad de vida de ellos. Diversos métodos paliativos como quimio y radioterapia han sido efectivos pero acompañados de significativos efectos colaterales y complicaciones ${ }^{14,18,19}$.

Actualmente, el rol de la cirugía de bypass con colon o estómago retro esternal es indicado excepcionalmente y sólo reservado a pacientes en muy buenas condiciones y con expectativa de sobrevida 
mayor de 1 año, pero en general esta práctica no es la mejor opción por su riesgo de morbimortalidad elevada siendo su rol en la era de la terapia endoluminal muy limitada, habiendo sido prácticamente abandonada ${ }^{13}$.

La radioterapia externa, acompañada o no con otras terapias, ha sido ampliamente difundida como opción terapéutica paliativa, pero se acompaña de estenosis fibrosa por lo que se tiene que asociar a dilataciones. La quimioterapia sola o en combinación con radioterapia no ha mostrado mejores resultados y presenta hasta un $40 \%$ de efectos colaterales. Respecto de las otras opciones como las dilataciones con bujías de Savary como medida paliativa, aparte de tener siempre un riesgo de perforación, la mejoría de la disfagia es corta y debe someterse a dilataciones periódicas ${ }^{13-15,17}$. La colocación de una prótesis es un procedimiento definitivo muy útil frente a la complicación. La alcoholización tumoral se ha usado en combinación con las dilataciones ${ }^{20}$ y no hay experiencia en nuestro departamento con braquiterapia o tunelización con láser. Por estas razones es que, al igual como muchos otros centros dedicados al tema, hemos optado por la colocación de prótesis transtumoral como tratamiento paliativo del cáncer esofágico irresecable ${ }^{21,22}$.

Existe una amplia variedad de prótesis disponibles en el mercado. Inicialmente eran de plástico para ser usadas por vía quirúrgica, pero actualmente no se usan, pues presentaban complicaciones y eran de difícil manejo, por lo que han dejado su lugar a las prótesis metálicas auto expansibles recubiertas ${ }^{23}$. En algunos casos se necesita de al menos una dilatación previa para lograr un lumen necesario para introducir el endoscopio y luego asegurar la correcta posición de la prótesis ${ }^{22}$. Por otra parte, las prótesis metálicas auto expansibles hoy son más fáciles de obtener, de diseño más simple y de más fácil colocación, a un costo más razonable que años anteriores. Permiten una hospitalización corta de 24-48 h. Presentan baja tasa de complicaciones y la mortalidad relacionada va de 3,4 a 16,8\%. La complicación más grave es la perforación, que puede presentarse hasta en un 6\% de los casos, lo que se asocia a una mortalidad de entre un $30-50 \% \%^{24,25}$. Los resultados obtenidos en nuestra experiencia respecto al uso de prótesis auto expansible para paliación son muy similares a lo publicado en la literatura, con un alto porcentaje de éxito en la colocación de la prótesis y mínima mortalidad en relación al procedimiento; las complicaciones son casi las mismas reportadas en la literatura ${ }^{17,23}$.

Las complicaciones tardías más frecuentes incluyen el desplazamiento (12\%) y la obstrucción de la prótesis por alimentos impactados o por crecimiento de la neoplasia $(3,4 \%-10 \%)^{22-26}$. Otras compli- caciones son la incapacidad de auto expansión o complicaciones respiratorias y compresión de la vía aérea que se presentan en un grado variable según el modelo de prótesis ${ }^{17}$. Estas complicaciones han sido observadas también en nuestra experiencia.

En general las indicaciones específicas merecen algunos comentarios:

\section{a) En neoplasias del tercio superior hay 2 puntos a observar}

En primer lugar tener un espacio suficiente bajo el nivel del cricofaríngeo para no tener molestias intratables debidas a la prótesis por irritación local, sensación de cuerpo extraño, tos crónica, etc. y en segundo lugar observar la relación con la vía aérea para evitar compresión la que puede ser advertida ya en el estudio de imágenes.

En fístulas esófago traqueales o bronquiales se piensa que es la mejor opción de paliación pues todas las otras alternativas de manejo, o perpetúan la fistula o las pueden incluso agravar con la alcoholización, dilatación, radioterapia o láser ${ }^{27,28}$.

Para los tumores de la unión gastroesofágica, el riesgo de torsión excesiva o de desplazamiento son más frecuentes en esta localización.

\section{b) Cáncer de tercio medio totalmente obstructivos}

Muchos radiólogos o cirujanos no endoscopistas consideran inapropiada la colocación, sin embargo, si es posible pasar un alambre guía y luego dilatar progresivamente es posible la inserción de la prótesis ${ }^{29}$.

Los resultados actuales respecto de la colocación de prótesis trans-tumorales por vía endoscópica aparecen técnicamente factibles, teniendo éxito en el tratamiento de la disfagia secundaria a tumores esofágicos $\mathrm{y}$ gastroesofágicos ${ }^{15}$. Estos últimos pacientes pueden presentar frecuentemente reflujo gastroesofágico ${ }^{21}$.

Nuestros estudios han combinado la colocación de prótesis con quimioterapia para lograr mayores sobrevidas ${ }^{24}$.

En el presente estudio, se han confirmado los resultados repetidos en la literatura, dando cuenta de un $100 \%$ de éxito en la colocación de prótesis y resultados clínicos satisfactorios cercanos al $90 \%$, con escasa morbilidad y sin mortalidad derivada de la técnica. Las complicaciones derivadas del reflujo gastroesofágico son eficazmente tratadas en estos pacientes, tanto con inhibidores de la bomba de protones, como prótesis especiales con dispositivos antirreflujo. Sin embargo, este es un punto aún controversial ${ }^{23,29,30}$.

Respecto de las complicaciones reportadas, se resumen en la Tabla 7, de ellas destacan la migración y la recurrencia de la disfagia. La tasa de reinterven- 
ción por esta causa en previas publicaciones varían entre $21 \%$ y $36 \%{ }^{28,31-34}$.

La migración del stent varía entre un $10,5 \%$ a $37 \%$, variando por tipo de prótesis usada, plástica o metálica auto expandible; las prótesis recubiertas presentan porcentaje de migración entre un $7 \%$ y $50 \%$.

Respecto de la hemorragia secundaria a la prótesis, en la literatura se señalan hasta en un $8 \%$, tanto para prótesis auto expandibles, como metálicas ${ }^{28}$.

La disfunción protésica causante de recurrencia de la obstrucción y disfagia ocurren en un $32,9 \% \%^{23,30}$, es secundaria a crecimiento tumoral por encima de la prótesis o trans-protésica, atascamiento de alimento, o migración. La obstrucción por crecimiento tumoral trans-protésica, se observa más frecuentemente en prótesis no recubiertas mientras que la migración es más frecuente en las prótesis recubiertas. Otros autores opinan que esta complicación no debiera ocurrir, porque si bien hay disminución de la estenosis tumoral, se produce edema, luego fibrosis y aperistalsis, que mantiene la prótesis en esta posición. La media de permeabilidad de las prótesis publicadas en la literatura, es de aproximadamente 6 meses, similar a nuestros resultados, la que es posible prolongar con quimio-radioterapia, pero tiene el agravante de favorecer la migración, debido a la disminución del tamaño o grado de estenosis tumoral $1^{23,26-31}$.

Mejores estudios se necesitan para dilucidar esta discusión.

En conclusión, de acuerdo a la experiencia reportada y a la propia experiencia local es posible plantear que las prótesis auto expansibles plásticas o metálicas actualmente son probablemente la mejor opción para el tratamiento paliativo del cáncer esofágico o de la unión gastroesofágica irresecables. La colocación de este tipo de prótesis auto expandibles, es factible y otorga un efectivo tratamiento de la disfagia en pacientes con cáncer esofágico y de la unión gastroesofágica, lo que concuerda con estudios internacionales.

\section{Referencias}

1. Datos estadísticos Minsal 2010 y 2011. www.minsal.cl

2. Hurschel JB. Van Sandick JW, de Boer AG, Wijnhoven BP, Tijssen JG, Fockens P, et al. Extended transthoracic resection compared with limited transhiatal resection for adenocarcinoma of the esophagus. N Engl J Med. 2002:347:1662-9.

3. Pisani P, Parkin DM, Bray F, Ferlay J. Estimates of the worldwide mortality from 25 cancer in 1990 Int J Cancer 1999.83:18-29.

4. Sjoquist KM, Burmeister BH, Smithers BM, Zalcberg
JR, Simes RJ, Barbour A, et al. Australasian GastroIntestinal Trials Group. Survival after neoadjuvant chemotherapy or chemoradiotherapy for resectable oesophageal carcinoma: an updated meta-analysis. Lancet Oncol. 2011;12:681-92.

5. Amdal CD, Jacobsen AB, Sandstad B, Warloe T, Bjordal K. Palliative brachytherapy with or without primary stent placement in patients with oesophageal cancer, a randomised phase III trial. Radiother Oncol. 2013;107:428-33.

6. Malthaner R, Fenlon D. Preoperative chemotherapy for resectable thoracic esophageal cancer. Cochrane Database Syst Rev. 2003;4:CD001556.

7. Sundelof M, Ye W, Dickman PW, Lagergren J. Improved survival in both histological types of esophageal cancer in Sweden. Int J Cancer 2002;99:751-54.

8. Homs M, Essink-Bot ML, Borsboom G, Steyerberg E, Siersema PD, Dutch SIREC Study Group. Quality of life after palliative treatment for esophageal carcinomaa prospective comparison between stent placement and single dose brachyterapy. Europ $\mathrm{J}$ of Cancer 2004;40:1862-71.

9. Dikken JL,van Sandick JW, Allum WH, Johansson J, Jensen LS, Putter H, et al. Differences in outcomes of esophageal and gastric cancer surgery across Europe. $\mathrm{Br}$ J Surg. 2013;100:83-94.

10. Ruol A, Castoro C, Portale G, Cavallin F, Sileni VC, Cagol M, et al. Trends in management and prognosis for esophageal cancer surgery: twenty-five years of experience at a single institution. Arch Surg. 2009;144:24754.

11. Dubecz A, Molena D, Peters JH. Modern surgery for esophageal cancer. Gastroenterol Clin North Am. 2008; 37:965-87.

12. Braghetto I, Csendes A, Cardemil G, Burdiles P, Korn $\mathrm{O}$, Valladares $\mathrm{H}$. Open transthoracic or transhiatal esophagectomy versus minimally invasive esophagectomy in terms of morbidity, mortality and survival. Surg Endosc. 2006;20:1681-6.

13. Ruiz F, Llanos O, Ibáñez L, Guzmán S, Llanos O, Pons A. Cáncer de esófago. Rev Chil Cir. 1995;47:437-43.

14. Braghetto I, Amat J, Rebolledo P, Ferrario M. Tratamiento paliativo del cáncer de esófago irresecable. Rev Chil Cir. 1994:46:377-84.

15. Rodríguez A, Moreno S, Gemmato AM, Anselmi M. Resultados del uso de prótesis metálicas auto expansibles tipo Choostent ${ }^{\circledR}$ en la paliación de pacientes con estenosis, fístulas o fugas esofágicas secundarias a patología maligna del esófago y/o cardias. Libro de resúmenes LXXXII Congreso Chileno de Cirugía 2009.

16. Braghetto I, Rebolledo P, Csendes A, Braghetto H. Confección y colocación de prótesis endoluminal para cáncer de esófago y cardias. Rev Chil Cir. 1990;42:314.

17. Braghetto I, Watkins G, Díaz L, Muñoz P. Complicaciones por dilatación endoscópica en patología benignas y 
malignas del esófago. Gastroenterol Latinoam. 1995;6 (2Suppl):52.

18. Warren W. Esophageal Cancer. Palliation of dysphagia. Chest Surg Clin NAm. 2000;10:605-23.

19. Barr H, Krasner N, Raouf A, Walker RJ. Prospective randomized trial of laser therapy only and laser therapy followed by endoscopic intubation for the palliation of malignant dysphagia. Gut 1990;31:252-8.

20. Moreira LS, Coelho RC, Sadala RU, Dani R. The use of ethanol injection under endoscopic control to palliate dysphagia caused by esophagogastric cancer. Endoscopy 1994;26:311-4.

21. Schoppemeyer K, Golsong J, Schiefke I, Mössner J, Caca K. Antireflux stent for palliation of malignant esophagocardial stenosis. Disease Esoph. 2007;20:8993.

22. Ramírez FC, Dennert B, Zierer ST, Sanowski RA. Esophageal self expanding metallic stents: Indications, practice techniques and complications. Results of a national study. Gastroeintestinal Endosc. 1997;45:360-4.

23. Rollhauser C, Fleischer DE. Late migration of a self expanding metal stent and successful endoscopic Management. Gastrointest Endosc. 1999;49:541-4.

24. Park JJ, Lee YC, Kim BK, Kim JH, Park JC, Kim YJ, et al. Long term clinical outcomes of self-expanding metal stents for treatment of malignant gastro esophageal junction obstructions and prognostic factors for stent patency: effects of anticancer treatments. Digestive and Liver Disease 2010;42:436-40.

25. Kirberg A. Cáncer de Esófago: Tratamiento endoscópico paliativo. Gastroent Latinoam. 2005;16:170-3.

26. Anselmi M, Gemmato P, Rodríguez A. Prótesis Choos- tent como método de paliación de la disfagia secundaria a patología maligna. Rev Chil Cir. 2012;64:546-54.

27. Adler D, Fang J, Wong R, Wills J, Hilden K. Placement of Poliflex stents in patients with locally advanced esophageal cancer is safe and improves dysphagia during neoadjuvant therapy. Gastrointest Endosc. 2009;70:614-9.

28. Bethge N, Vakil N. A prospective trial of a new selfexpanding plastic stent for malignant esophageal obstruction. Am J Gastroent. 2001;96:1350-4.

29. Radecke K, Gerken G, Treichel U. Impact of a self-expanding, plastic esophageal stent on various esophageal stenosis, fistulas and leakages: a single-center experience in 39 patients. Gatrointest Endosc. 2005;61: 812-8.

30. Baron TH. Minimizing endoscopic complications. Endoluminal stents. Gastrointest Endosc Clin N Am. 2007; 17:83-104.

31. Homs MY, Wabab PJ, Kuipers EJ, Steyerberg EW, Grool TA, Haringsma J. Esophageal stents with antireflux valve for tumors of the distal esophagus and gastric cardia: a randomized trial. Gastrointest Endosc. 2004;60:695-702.

32. Simmons DT, Baron TH. Endoluminal palliation. Gastrointest endosc. Clin N Am. 2005;15:467-84.

33. Dormann AJ, Eisendrath P, Wigginhaus B, Huchzermeyer H, Deviere J. Palliation of esophageal carcinoma with a new self-expanding plastic stent. Endoscopy 2003;35:207-11.

34. Costamagna G, Shah SK, Tringali A, Mutignani M, Perri V, Riccioni ME, Prospective evaluation of a new self expanding plastic stent for inoperable esophageal strictures. Surg Endosc. 2003;17:891-5. 